\title{
TELEOFTALMOLOGIA COMO AUXÍlIO DIAGNÓSTICO NAS DOENÇAS INFECCIOSAS E INFLAMATÓRIAS OCULARES
}

\author{
luciana Peixoto dos Santos finamor*, Maria Cristina Martins, Cristina Muccioli, Daniel Singulem, Paulo R. L. Lopes, Rubens Belfort Jr \\ Trabalho realizado no Departamento de Oftalmologia e Departamento de Informática em Saúde da UNIFESP, São Paulo, SP.
}

\author{
*Correspondência: \\ Universidade Federal de \\ São Paulo - UNIFESP \\ R. Botucatu, 824 \\ Vila Clementino, São Paulo, \\ SP, Fone: (11) 5085-2027. \\ lucpeixoto@aol.com
}

\begin{abstract}
RESUMO
OBjetivos. Avaliar a eficácia da teleoftalmologia como auxilio diagnóstico nas doenças infecciosas e inflamatórias oculares, assim como avaliar os principais fatores limitantes para a realizaçãa e conclusão de consultoria em oftalmologia.

Métodos. Duzentas e trinta e três fotos (retinografias e/ou exames de biomicroscopia), em diapositivos 35-mm de 87 pacientes, foram digitalizadas e armazenadas em CD-ROM, para posterior análise, em monitor de computador, por dois consultores especialistas em uveítes, que responderam formulário e questionário indicando o diagnóstico provável, tratamento e/ou conduta e as dificuldades encontradas para a conclusão da consultoria. Teste estatístico Kappa foi utilizado para avaliação da concordância entre os observadores. Valores de Kappa acima de 0,7 indicam concordância excelente; valores entre 0,4 e 0,7 indicam concordância moderada/boa e valores menores que 0,4 indicam concordância ruim.

REsultados. A concordância diagnóstica observada na consultoria, em relação ao padrão ouro, foi de $73,5 \%$ para ambos os consultores. Sugestão de tratamento e/ou conduta não foi possível em 8\% dos casos para o consultor A, e em 10,4\% para o consultor B. A falta de dados clínicos (Kappa: 0,8) e a má qualidade das imagens (Kappa: 0,74) foram os motivos com maior concordância em relação às dificuldades para conclusão da consultoria.

Conclusão. A teleoftalmologia por meio de consultoria por método assíncrono foi eficaz para o diagnóstico de doenças infecciosas e inflamatórias oculares. A falta de dados clínicos detalhados e a má qualidade das imagens foram os principais fatores limitantes para a conclusão da consultoria.
\end{abstract}

Unitermos: Telemedicina. Oftalmologia. Diagnóstico. Uveítes. Síndrome de imunodeficiência adquirida.

\section{INTRODUÇÃO}

Grandes distâncias entre os centros médicos especializados e os locais mais carentes, além de recursos escassos e mal distribuídos, representam dificuldades na área da saúde que podem ser diminuídas com a telemedicina!. Telemedicina é definida como a oferta de serviços ligados aos cuidados com a saúde, nos casos em que a distância é um fator crítico. Tais serviços são oferecidos por profissionais da área de saúde, usando tecnologias de informaçã̃o e de comunicação, visando o intercâmbio de informações válidas para pesquisas, diagnósticos, prevenção e tratamento de doenças e contínua educação de provedores de cuidados com a saúde, no interesse de melhorar a saúde das pessoas e de suas comunidades².

Praticamente quase todas as especialidades médicas podem vir a utilizar-se da telemedicina, particularmente aquelas que utilizam imagens como meio diagnóstico. Assim, os setores de radiologia, dermatologia, patologia, ultra-sonografia, oftalmologia, entre outros, são bastante propícios para o estabelecimento de protocolos de transmissão de dados à distância, com finalidades diagnósticas 3,4.

Existem três formas distintas de interação via telemedicina: a forma síncrona ou interativa (sistemas em tempo real), a forma assíncrona (sistemas de armazenamento e envio) e a híbrida, ${ }^{5,6,7}$.

No sistema síncrono, a comunicação entre dois ou mais indivíduos é feita em tempo real, na maioria das vezes por meio de videoconferência, embora até mesmo a consultoria via telefone possa ser considerada como forma interativa. Esse sistema permite consulta direta e provê opinião ou diagnóstico remoto imediato. $\mathrm{O}$ paciente, assistente primário e especialista têm que estar presentes simultaneamente, permitindo maior interação e efeito educacional ${ }^{8}$.

$\mathrm{Na}$ forma assíncrona, arquivos eletrônicos de áudio, texto, vídeo e/ou imagens são transmitidos para dispositivos remotos de armazenamento de dados, onde ficam acessíveis para revisão e consulta. Não exige presença simultânea das partes envolvidas e os custos de transmissão são inferiores, porém a eficácia dessa interação depende da qualidade da informação fornecida pelo assistente primário de saúde?.

A eficácia diagnóstica da telepatologia ${ }^{10.13}$, telerradiologia ${ }^{14-17} \mathrm{e}$ teledermatologia ${ }^{18-22}$, por meio da análise de fotos ou imagens armazenadas, já foi demonstrada em vários estudos.

Weinberg et al. ${ }^{10}$ avaliaram a eficácia da telepatologia comparando imagens digitais armazenadas, com o exame convencional. A concordância encontrada entre os dois métodos foi de 87,5\%. Weinstein \& Epstein ${ }^{23}$ avaliaram biópsias de próstata, com concordância de $90,5 \%$ e Singson et al. ${ }^{24}$ obtiveram concordância de $94 \%$ em imagens digitalizadas de biópsias gastrintestinais.

A concordância entre patologistas, em relação a diagnóstico de biópsias de pele, foi estudada por Piccolo et al..$^{25}$. Nesse estudo, patologistas realizaram teleconsulta, usando o método assíncrono (análise de imagens recebidas por e-mail), sendo que o diagnóstico foi correto em 78\% dos casos. 
FINAMOR LPS ET AL.

Molnar et al. ${ }^{26}$ utilizaram a telepatologia para diagnóstico de biópsias gastrintestinais, sendo que os principais motivos citados como causas de discordância entre os observadores foram a qualidade da foto, diferença na interpretação da imagem e informação clínica insuficiente.

Chao et al. ${ }^{22}$ descreveram um sistema de teledermatologia via internet, e compararam a concordância entre o diagnóstico virtual, por meio da avaliação de imagens armazenadas com o diagnóstico "face a face", encontrando concordância de $95 \%$ entre os dois métodos.

A teleoftalmologia representa um setor emergente, caminhando para a maturidade. É uma área que pode possibilitar diagnóstico, tratamento, pesquisa, rastreamento de doenças, segunda opinião e educação médica continuada ${ }^{27}$.

Triagem, avaliação e tratamento de pacientes com retinopatia diabética são algumas das aplicações mais populares da teleoftalmologia, por meio da análise de imagens obtidas por câmeras de retina portáteis, em lugares remotos ${ }^{28-33}$. Klein et al. ${ }^{28}$ encontraram concordância de $82,5 \%$ no diagnóstico de retinopatia diabética, realizado por meio desta câmera, quando comparado com aquele feito com fotos estereoscópicas de filme tradicional. Gómez-Ulla et al. ${ }^{31}$ avaliaram imagens digitais de pacientes diabéticos, enviadas por internet, encontrando concordância de 100\% para casos com presença ou ausência de descolamento de retina. A concordância para os outros achados foi de $94 \%$.

Poucos estudos fazem referência ao uso da telemedicina em pacientes com doenças infecciosas e inflamatórias oculares. A possibilidade de diagnóstico, via telemedicina, da retinite pelo citomegalovírus (CMV) foi estudada por Li et al. em 30 olhos $^{34}$. A concordância entre os métodos foi de 100\%.

Apesar dos possíveis benefícios, a teleoftalmologia ainda não é utilizada no Brasil, e a eficácia diagnóstica de serviços de consultoria de oftalmologia, via telemedicina, não é determinada.

\section{Objetivos}

I. Avaliar a eficácia de método assíncrono de consultoria em oftalmologia no diagnóstico das doenças infecciosas e inflamatórias oculares;

2. Avaliar os principais fatores limitantes para a realização e conclusão de consultoria via teleoftalmologia.

\section{MÉtodos}

Após aprovação de protocolo pelo comitê de ética em pesquisa da Universidade Federal de São Paulo -UNIFESP, 233 fotos (retinografias e/ou exames de biomicroscopia, em diapositivos 35-mm) de 87 pacientes, do arquivo de fotos do setor de Uveítes e AIDS do departamento de oftalmologia da UNIFESP/EPM foram aleatoriamente selecionadas, digitalizadas (Scanner Kodak 3600), comprimidas (2:1) em arquivo tipo JPEG (Joint Photographic Expert Group) e armazenadas em CD-ROM, para posterior análise em monitor de computador por dois observadores (A e B), especialistas em doenças infecciosas e inflamatórias oculares.

Os observadores (consultores) receberam, para análise, CD-ROM com as imagens armazenadas e formulário de consultoria, com dados retirados dos prontuários de tais pacientes, abrangendo: anamnese, acuidade visual, exames laboratoriais disponíveis, antecedentes pessoais e familiares. Durante o estudo, não houve comunicação entre os observadores no que se refere à análise das imagens. Os consultores, após avaliação das imagens, responderam a questionário específico, nos quais se avaliou:

- motivos que dificultaram ou impossibilitaram a conclusão da consultoria: necessidade de fotos de outros campos, necessidade de fotos do olho contralateral, má qualidade da foto, opacidade de meios, imagem mal definida, falta de dados clínicos, falta de exames laboratoriais e magnificação;

- possibilidade de sugestão diagnóstica com a consultoria;

- possibilidade de sugestão de tratamento e/ou conduta, baseada nos dados analisados.

Os resultados obtidos na consultoria foram avaliados por um terceiro observador $\mathbf{C}$ (considerado padrão ouro), que tinha acesso aos diapositivos selecionados e aos prontuários dos pacientes, com conhecimento do diagnóstico correto ou provável, previamente determinado pelo setor de Uveítes e AIDS do departamento de oftalmologia da UNIFESP-EPM. O grau de dificuldade dos casos foi previamente determinado pelo observador $\mathbf{C}$, baseado nos dados apresentados no prontuário médico e nas características da manifestação clínica apresentada.

A concordância das respostas dos observadores, bem como os principais fatores determinantes da impossibilidade do diagnóstico, foram avaliados.

\section{Análise estatística}

Teste estatístico Kappa (k) foi utilizado para avaliação de concordância entre os consultores. O teste $\mathrm{K}$ avalia se houve concordância nas respostas, acima da que seria esperada ao acaso, e a reprodutibilidade dos resultados.

Fleiss $^{35}$ recomenda que valores de Kappa acima de 0,7 indiquem concordância excelente; valores entre 0,4 e 0,7 concordância moderada/boa e valores menores que 0,4 concordância ruim.

O teste de McNemar foi utilizado para avaliação do grau de discordância entre os observadores, adotando-se como significante $p<0,05$.

\section{Resultados}

Duzentas e trinta e três imagens digitalizadas, de 87 pacientes, foram avaliadas pelos consultores $\mathrm{A}$ e $\mathrm{B}$. A média de imagens analisadas em cada consultoria foi de 2,7 fotos. Cinqüenta e sete por cento dos casos eram pacientes com AIDS. Segundo avaliação do observador C, em 33,3\% dos casos o diagnóstico foi considerado fácil, em $34,5 \%$, de média dificuldade e em $32,2 \%$, difícil. Em 12 casos ( I3,8\%), o diagnóstico foi inconclusivo, só sendo possível o estabelecimento de diagnóstico sindrômico e/ou etiológico provável, pelo padrão ouro.

Em relação aos diagnósticos etiológicos do consultor $\mathrm{A}$, em seis casos (6,8\%) a sugestão foi considerada errada, sendo que cinco desses casos (93\%) foram previamente classificados como sendo de difícil diagnóstico pelo padrão ouro. Em 19,5\% dos casos (17 casos), o consultor A indicou mais de uma etiologia provável (diagnóstico 
inconclusivo), sendo que em todos esses casos um dos diagnósticos foi o diagnóstico considerado pelo padrão ouro. Oito destes casos (47\%) haviam também recebido, previamente, diagnóstico inconclusivo pelo observador C (padrão ouro).

Em 73,5\% dos casos (64 casos), o diagnóstico do consultor $A$ foi o mesmo apresentado pelo padrão ouro.

Em relação aos diagnósticos etiológicos do consultor $B$, em dois casos (2,3\%), a sugestão foi considerada errada, sendo que um desses casos foi previamente classificado como sendo de difícil diagnóstico e o outro, como de média dificuldade diagnóstica. Em $24 \%$ dos casos (2I casos), o consultor B indicou mais de uma etiologia provável como diagnóstico da consultoria (diagnóstico inconclusivo), sendo que em todos esses casos um dos diagnósticos foi o diagnóstico considerado pelo padrão ouro. Onze (55\%) desses casos foram, também, os casos com mais de um diagnóstico etiológico citado, considerados pelo consultor $A$, sendo que oito deles (38\%) haviam também recebido, previamente, diagnóstico inconclusivo pelo observador C (padrão ouro).

Em 73,5\% dos casos (64 casos), o diagnóstico do consultor B foi o mesmo apresentado pelo padrão ouro. Em 71,3\% dos casos o diagnóstico do consultor $A$ foi igual ao do consultor $B$ (casos com apenas uma indicação de diagnóstico) e, em 93,1\% dos casos (8I casos), pelo menos um dos diagnósticos sugeridos foi o mesmo para os dois consultores ( $\mathrm{A}$ e B). Não houve nenhum caso em que o diagnóstico sugerido tenha sido incorreto, pelos dois consultores.

A Tabela I e a Figura I apresentam a concordância entre os consultores $A$ e $B$ em relação ao diagnóstico Etiológico, sugestão de tratamento e/ou conduta e grau de dificuldade na consultoria. Observa-se concordância excelente em relação a diagnóstico etiológico e sugestão de tratamento e/ou conduta (Kappa >0,7) e concordância moderada/boa para grau de dificuldade (Kappa: 0,51). 0 teste de McNemar para avaliação de discordância não foi significativo para todas as variáveis acima mencionadas $(p>0,05)$.

Em relação aos motivos que dificultaram ou impossibilitaram a consultoria, o maior nível de concordância, entre os consultores A e B, ocorreu nos parâmetros falta de dados clínicos e má qualidade da foto, com Kappa de 0,80 e 0,74, respectivamente, conforme demonstrado na Tabela 2. Os outros motivos apresentaram Kappa <0,4, apesar dos altos níveis de concordância observados (64,3\% a 83,9\%). O teste de McNemar não foi significativo para todos os parâmetros acima ( $p>0,05)$.

Sugestão de tratamento e/ou conduta ocorreu em 91,9\% das consultorias realizadas pelo consultor A e em $89,6 \%$ das consultorias realizadas pelo consultor $B$, sendo a diferença entre os dois consultores não significante $(p>0,05)$.

Os motivos que mais dificultaram a conclusão da consultoria foram falta de dados clínicos (35\% A e 39\% B), falta de exames laboratoriais (25\% A e 30\% B) e má qualidade da foto (I0\% A e B), sem diferença estatisticamente significante entre eles $(p>0,05)$.

\section{Discussão}

A teleoftalmologia é uma área emergente, principalmente no Brasil, onde estudos e publicações de âmbito nacional ainda não estão disponíveis na literatura. As dificuldades encontradas para implantação de um

\begin{tabular}{|c|c|c|c|}
\hline \multicolumn{4}{|c|}{$\begin{array}{l}\text { Tabela I - Concordância entre os consultores A e B, em relação a } \\
\text { diagnóstico etiológico, diagnóstico sindrômico, sugestão de } \\
\text { tratamento e/ou conduta e grau de dificuldade na consultoria }\end{array}$} \\
\hline Variável & Concordância & Kappa & McNemar \\
\hline Diagnóstico etiológico & $90,8 \%$ & $\overline{0,77}$ & 0,723 \\
\hline $\begin{array}{l}\text { Sugestão de tratamento } \\
\text { e/ou conduta }\end{array}$ & $95,4 \%$ & 0,72 & 0,617 \\
\hline Grau de dificuldade & $75,8 \%$ & 0,51 & 0,248 \\
\hline
\end{tabular}

Figura I - Gráfico demonstrativo do Kappa

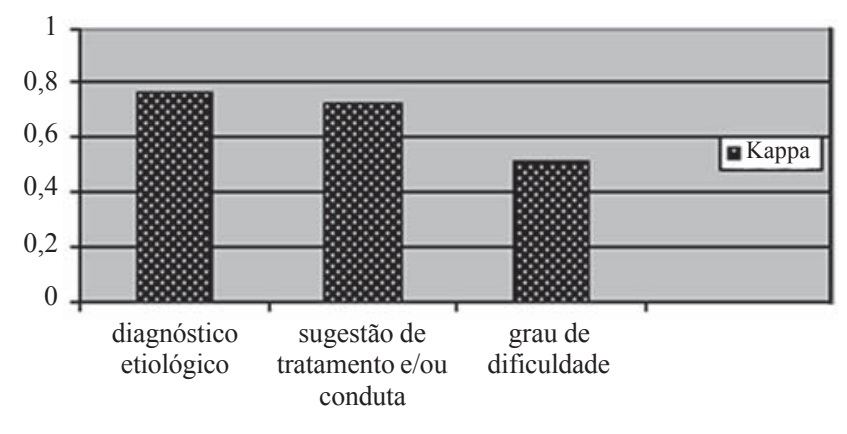

Tabela 2 - Concordância entre os consultores A e B em relação aos motivos que dificultaram ou impossibilitaram a consultoria

\begin{tabular}{|c|c|c|c|}
\hline Motivos da dificuldade & Concordância & Kappa & McNemar \\
\hline $\begin{array}{l}\text { Necessidade de fotos de } \\
\text { outros campos }\end{array}$ & $83,9 \%$ & 0,03 & 0,789 \\
\hline $\begin{array}{l}\text { Necessidade de fotos do } \\
\text { olho contralateral }\end{array}$ & $82,7 \%$ & 0,11 & 0,479 \\
\hline Má qualidade da foto & $93,1 \%$ & 0,74 & 1,000 \\
\hline Opacidade de meios & $83,9 \%$ & 0,21 & 0,181 \\
\hline Imagem mal definida & $85 \%$ & 0,07 & 0,096 \\
\hline Falta de dados clínicos & $90,8 \%$ & 0,80 & 0,723 \\
\hline Falta de exames laboratoriais & $64,3 \%$ & 0,27 & 0,719 \\
\hline Magnificação & $96,5 \%$ & 0,38 & 0,248 \\
\hline
\end{tabular}

serviço de teleoftalmologia podem ser muitas, sendo que a ausência de diretrizes e metas já pré-determinadas pode dificultar o desenvolvimento de projetos nesta área. A utilização da infra-estrutura de comunicação, já existente no local, pode representar uma alternativa para a implementação de um sistema de telemedicina ${ }^{36,37}$.

A disponibilidade, altamente difundida, das redes de computadores, bem como a rapidez na transmissão de dados, têm resultado na possibilidade, cada vez maior, de se obter diagnósticos via consultoria, a partir de pontos remotos. Com o aumento da popularidade desse meio de diagnóstico, é importante assegurar que a acurácia desse método se mantenha em níveis altos. Neste estudo, avaliamos a 
FINAMOR LPS ET AL.

utilidade da teleoftalmologia como auxílio diagnóstico nas doenças infecciosas e inflamatórias oculares, por meio de um sistema assíncrono de consultoria, realizada por dois especialistas.

A forma assíncrona é considerada mais eficiente que a forma síncrona (tempo real) para a análise de figuras estáticas, como as deste estudo, visto que imagens estáticas apresentam maior qualidade e resolução que imagens em movimento, transmitidas em tempo real, permitindo, assim, uma maior acurácia diagnóstica ${ }^{6,38}$. Não exige presença simultânea das partes envolvidas e, segundo alguns estudos, os custos de transmissão são inferiores s9,40. $^{39}$.

A principal desvantagem dessa forma é que ela depende da qualidade das informações fornecidas pelo assistente primário de saúde, sendo que informações inadequadas ou insuficientes poderiam prejudicar o resultado de uma consultoria?.

Vários estudos comparam a eficácia diagnóstica de sistemas de telemedicina assíncronos, com a encontrada no exame face a face e a variabilidade interobservadores em sistemas de teleconsultoria 13,22,24,25,41-45. No presente estudo, apuramos que a eficácia diagnóstica da consultoria para o consultor A foi de $73,5 \%$. Se considerarmos os casos em que mais de um diagnóstico diferencial foi citado, sendo pelo menos um deles o diagnóstico correto, esse número sobe para $93 \%$.

A eficácia diagnóstica da consultoria do consultor $\mathrm{B}$ foi igual à do consultor A (73,5\%). Se considerarmos os casos em que mais de um diagnóstico diferencial foi citado, sendo pelo menos um deles o diagnóstico correto, esse número sobe para $97,7 \%$.

A maioria dos estudos que avaliam a eficácia da telemedicina como método diagnóstico encontram resultados semelhantes ou inferiores ao método convencional, porém dentro de limites aceitáveis. A variabilidade de respostas interobservadores também esteve presente nesses estudos ${ }^{42-46}$.

A indicação de mais de uma etiologia, em alguns casos, pelos dois consultores, pode sugerir que a confiança do consultor em indicar um único diagnóstico, por meio da análise à distância de pacientes, pode ser menor. Briggs et al. ${ }^{41}$ avaliaram os efeitos da confiança dos examinadores na acurácia dos diagnósticos indicados por eles após avaliação de imagens digitalizadas ou em diapositivos. Segundo esse trabalho, as imagens digitalizadas causaram diminuição na confiança das respostas, porém alteraram pouco a acurácia diagnóstica dos mesmos.

Em relaçãa à sugestão de tratamento e/ou conduta, observou-se concordância excelente entre os dois consultores ( $k: 0,72)$, sendo que o consultor A sugeriu tratamento e/ou conduta em 91,9\% das consultorias, e o consultor B, em 89\% dos casos. A possibilidade de indicação de tratamento e/ou conduta, por meio de telemedicina, é fator fundamental na avaliação desses sistemas ${ }^{47}$.

Os motivos que mais dificultaram a conclusão da consultoria para os consultores A e B foram a falta de dados clínicos, falta de exames laboratoriais e má qualidade da foto, sem diferença estatisticamente significante entre os dois consultores $(p>0,05)$. O maior grau de concordância ocorreu nos itens falta de dados clínicos e má qualidade da foto, com Kappa de 0,8 e 0,74, respectivamente, o que indica concordância excelente e alta reprodutibilidade para esses parâmetros.

Os principais motivos que levariam à dificuldade na interpretação de uma teleconsultoria já foram avaliados por outros autores ${ }^{10,13,23,26}$. Segundo Baruffaldi et al. ${ }^{38}$, os principais problemas que afetam a telemedicina são a falta ou baixa qualidade das informações recebidas do local primário. De acordo com a Declaração de Tel Avivis, o médico teleconsultado não deve responder se não obteve suficiente informação do paciente ou mesmo do médico local para que possa dar uma opinião bem fundamentada.

Formulários de consultoria via telemedicina devem dar atenção especial ao campo referente aos dados clínicos do paciente em questão, com necessidade obrigatória do correto preenchimento do mesmo. A falta desses dados pode representar causa de impedimento ou dificultar a conclusão da consultoria.

A oftalmologia parece ser uma área bastante propícia para o desenvolvimento da telemedicina, já que é uma especialidade em que o diagnóstico por imagens pode ser feito em muitos casos ${ }^{49}$. Porém, neste estudo, a má qualidade das imagens foi um dos principais motivos citados como causa de dificuldade na consultoria. Métodos para uma melhor aquisição das imagens registradas deverão ser investigados; é possível que a melhora na qualidade das imagens possa causar um aumento da eficácia diagnóstica via teleoftalmologia.

A metodologia aqui apresentada permitiu a simulação de um sistema de consultoria em teleoftalmologia para o diagnóstico de pacientes com doenças infecciosas e inflamatórias oculares, por meio de método assíncrono. A forma assíncrona pode representar um método fácil e barato para prestação de consultoria, visto que essas imagens podem ser armazenadas e enviadas para um ou mais especialistas, por meio de recursos altamente difundidos como a internet e e-mail, ou até mesmo armazenadas em unidades de gravação, como CD-ROM ou disquetes, e posteriormente avaliadas em outra unidade de computador.

\section{Conclusões}

I. A teleoftalmologia, por meio da utilização de método assíncrono de consultoria, realizada por consultores especialistas, foi eficaz para o diagnóstico de doenças infecciosas e inflamatórias oculares, com alta concordância diagnóstica para os casos analisados (73\% para ambos os consultores).

2. Os principais motivos que dificultaram ou impossibilitaram a conclusão da consultoria foram a falta de dados clínicos (Kappa: 0,8) e a má qualidade da foto (Kappa: 0,74), concordância excelente entre os observadores.

\section{Conflito de interesse: não há.}

\section{SUMMARY}

Teleophthalmology as an aUXILIARY approach FOR the DIAGNOSIS OF INFECTIOUS AND INFLAMMATORY OCULAR DISEASES: EVALUATION OF AN ASYNCHRONOUS METHOD OF CONSULTATION.

PURPOSE. The purpose of this study was to evaluate the efficacy of teleophthalmology as an auxiliary approach for diagnosis of infectious and inflammatory ocular diseases and to study the main problems associated with the conclusion reached by consultation.

METHODS. Two hundred and thirty three $35 \mathrm{~mm}$ slides (retinography or biomicroscopy photos) were digitalized and stored in a Compact Disc to be later analyzed in a computer monitor by two consultants, specialists in uveitis. The consultants filled out a form and a questionnaire, indicating the 
diagnosis and probable treatment for the analyzed cases also the main difficulties found to reach a conclusion of the consultation. The Kappa statistic, an assessment of the change-corrected agreement (reproducibility) among consultants, was calculated. Kappa values of $>$ 0.7 indicated excellent agreement; values between 0.4 and 0.7 indicated fair to good agreement and values of $<0.4$ indicated poor agreement.

RESULTS. Agreement between the consultants and the final diagnosis was of $73.5 \%$. Treatment suggestion or management was not possible in $8 \%$ of the cases for consultant $A$ and in $10.4 \%$ for consultant $B$. The higher agreement rate among consultants, relating to the reasons that rendered diagnosis difficult, was found in relation to "lack of clinical data" and "low quality of images".

CONCLUSION. Teleophthalmology, through the asynchronous method, was an efficient means for diagnosis of patients with infectious and inflammatory ocular diseases. The main problems affecting the teleophthalmology consultation were lack of detailed clinical data and low quality of the images. [Rev Assoc Med Bras 2005; 5 I (5): 279-84]

KEY wORDS: Telemedicine. Ophthalmology. Diagnosis. Uveitis. AIDS.

\section{ReFERÊNCIAS}

I. Maceratini R, Sabbatini MER. Telemedicina: a nova revolução. Rev Informédica 1994; 1:5-9.

2. Organização Mundial de Saúde. Telemedicina: definições [online]. 1997. [citado 2003 fev 20]. Disponível em: http://www.unifesp.br/dis/set/ oquee.html

3. Stanberry B. Telemedicine: barriers and opportunities in the 2 I st century. J Intern Med 2000;247:6 I 5-28.

4. Krupinski E, Nypaver M, Poropatich R, Ellis D, Safwat R, Sapci H. Clinical applications in telemedicine/telehealth. Telemed J E Health 2002;8: 13-34.

5. Wallace S, Wyatt J, Taylor P. Telemedicine in the NHS for the millennium and beyond. Postgrad Med J 1998;74:721-8.

6. LiHK. Telemedicine and ophthalmology. Surv Ophthalmol 1999;44:6 I-72.

7. Loane M, Wootton R. A Review of guidelines and standards for telemedicine. J Telemed Telecare 2002;8:63-7I.

8. Lamminen H, Salminen L, Uusitalo H. Teleconsultations between general practioners and ophthalmologists in Finland. J Telemed Telecare | 999; 5: | | 8-2|.

9. Seabra ALR. Telemedicina. In: Pitta GBB, Castro AA, Burihan E, editores. Angiologia e cirurgia vascular: guia ilustrado [monografia online]. Maceió: UNCISAL/ECMAL; 2000 [citado 2003 mar 15]. Disponível em: http:// www.lava.med.br/livro

10. Weinberg DS, Allaert FA, Dussere P. Telepathology diagnosis by means of digital still images: an international validadion study. Hum Pathol 1996;27: | | |-8.

1 I. Weinstein RS. Static image telepathology in perspective. Hum Pathol 1996;27:99-101.

12. Halliday BE, Bhattacharyya AK, Graham AR, Davis JR. Diagnostic accuracy of an international static-imaging telepathology consultaion service. Hum Pathol | 997;28: 17-21

13. Odze RD, Goldblum J, Noffsinger A, Alsaigh N, Rybicki LA, Fogt F. Interobserver variability in the diagnosis of ulcerative colitis - associated dysplasia by telepathology. Mod Pathol 2002; 1 5:379-86.

14. Scott WW, Bluemke DA, Mysko WK. Interpretation of emergency department radiographs by radiologists and emergency medicine physicians: teleradiology workstation versus radiograph readings. Radiology 1995; 197:223-9.

15. Elam EA, Rehm K, Hillman BJ, Maloney K. Efficacy of digital radiography for the detection of pneumothorax: comparision with conventional chest radiography. Am J Radiol 1992; | 58:509- 14.
16. Parasyn A, Hanson RM, Peat JK, De Silva M. A comparison between digital images viewed on a picture archiving and communication system diagnostic workstation and on a PC-based remote viewing system by emergency physicians. J Digit Imaging 1998; I I:45-9.

17. Eng J, Mysko Wk, Weller GE, Renard R, Gitlin JN. Interpretation of emergency department radiographs: a comparison of emergency medicine physicians with radiologists, residents with faculty, and film with digital display. Am J Radiol 2000; I 75: I 233-8.

18. Perednia DA, Gaines JA, Rossum AC. Variability in physician assessment of lesions in cutaneous images and its implications for skin screening and computer - assisted diagnosis. Arch Dermatol 1992; 128:257-64.

19. Barnard CM, Goldyne ME. Evaluation of an asynchronous teleconsultation system for diagnosis of skin cancer and other skin disease. Telemed J 2000;6:379-83.

20. Loane MA, Bloomer SE, Corbett R, Eedy DJ, Hicks N, Lotery C, et al. A comparison of real- time and store-and-forward teledermatology: a costbenefit study. Br J Dermatol 2000b; | 43: | 24|-7.

21. Wootton R, Bloomer SE, Corbett R, Eedy DJ, Hickis N, Lotey HE, et al. Multicentre randomized control trial comparing real time teledermatology with conventional outpatient dermatologic care: societal cost-benefit analysis. BMJ 2000;320: I 252-6.

22. Chao LW, Cestari TF, Bakos L, Oliveira MR, Miot HA, Zampese GM. Evaluation of an Internet-based teledermatology system. J Telemed Telecare 2003;9(Suppl I):S9- 12.

23. Weinstein $\mathrm{MH}$, Epstein JI. Telepathology diagnosis of prostate needle biopsies. Hum Pathol 1997;28:22-9.

24. Singson RPC, Natarajan S, Greenson J. Virtual microscopy and the Internet as telepathology consultation tools. A study of gastrointestinal biopsy specimen. Am J Clin Pathol 1999; I 1:729-5.

25. Piccolo D, Soyer HP, Burgdorf W, Talamini R, Peris K, Buggati L, et al. Concordance between telepathologic diagnosis and conventional histopathologic diagnosis: a multiobserver store-and-forward study on 20 skin specimens. Arch Dermatol 2002; 138:53-8.

26. Molnar B, Berczi L, Diczhazy C, Tagscherer A, Varga SV, Szende B, et al. Digital slide and virtual microscopy based routine and telepathology evaluation of routine gastrointestinal biopsy specimens. J Clin Pathol 2003;56:433-8.

27. Cuzzani O. Teleoftalmología, presente y futuro. Arch Soc Esp Oftalmol $2000 ; 1: 1-3$.

28. Klein R, Klein BEK, Neider MW. Diabetic retinopathy as detected using ophthalmoscopy, a nonmydriatic camera and standart fundus camera. Ophthalmology 1985;92:485-91.

29. Liesenfeld B, Kohner E, Piehlmeier W, Kluthe S, Aldington S, Hepp KD. A telemedical approach to the screening of diabetic retinopathy by digital fundus photography. Diabetes Care 2000;23:345-8.

30. Bursell SE, Cavallerano JD, Cavallerano AA, Clermont AC, Aiello LM; Joslin Vision Network Reaserch Team. Stereo nonmydriatic digital-video color retinal imaging compared with Early Treatment Diabetic Retinopathy Study seven standard field 35-mm stereo color photos for determining level of diabetic retinopathy. Ophthalmology 200 I; 1 08:572-85.

31. Gómez-Ulla F, Fernandez MI, Gonzalez F, Rey P, Gude F. Digital retinal images and teleophthalmology for detecting and grading diabetic retinopathy. Diabetes Care 2002;25: 1384-89.

32. Lin DY, Blumenkranz MS, Brothers RJ, Grosvenor DM. The sensitivity and specificity of single-field nonmydriatic monochromatic digital fundus photography with remote image interpretation for diabetic retinopathy screening: a comparison with ophthalmoscopy and standardized mydriatic color photography. Am J Ophthalmol 2002; I 34:204- I3.

33. Cavallerano AA, Cavallerano JD, Katalinic P, Tolson AM, Aiello LP, Aiello LM; Joslin Vision Network Clinical Team. Use of Joslin Vision Network digital-video nonmydriatic retinal imaging to assess diabetic retinopathy in a clinical program. Retina 2003;23:2 I5-23.

34. Li HK, Temprano J, Horna J, Tang R. Diagnosing cytomegalovirus retinitis through teleophthalmology. Internet J Ophthalmol Vis Sci [serial online] 2000;I(I)[I7 telas] [cited 2003 jul 2I]. Available from: http:// www.ispub.com/ostia/index.php?xmIPrinter $=$ true $\& \times m \mid F i l e P a t h$ $=$ journals/ijovs/vol InI/retinitis.xml 
FINAMOR LPS ET AL.

35. Fleiss JL. Statistical methods for rates and proportions. 2nd ed. New York: Wiley; 1981.

36. Wechsler R, Ancao MS, de Campos CJ, Sigulem D. Computing in medical practice. J Pediatr 2003;79(Suppl I):S3- I2.

37. Lamminen $H$, Voipio $V$, Ruohonen $K$, Uusitalo $H$. Telemedicine in ophthalmology. Acta Ophthalmol Scand 2003;81 : I 05-9.

38. Baruffaldi F, Gualdrini G, Toni A. Comparison of asynchronous and realtime teleconsulting for orthopaedic second opinions. J Telemed Telecare 2002;8:297-30I .

39. Jaatinen PT, Forsstrom J, Loula P. Teleconsultations: who uses them and how? Telemed Telecare 2002;8:319-24.

40. Loane MA, Bloomer SE, Corbett R, Eedy DJ, Hicks N, Lotery HE, et al. $A$ randomized controlled trial to asses the clinical effectiveness of both realtime and store-and-forward teledermatology compared with conventional care. J Telemed Telecare 2000;6(Suppl I):SI-3.

4I. Briggs R, Bailey JE, Eddy C, Sun I. A methodologic issue for ophthalmic telemedicine: image quality and its effect on diagnostic accuracy and confidence. J Am Optom Assoc 1998;69:60 I-5.

42. Nitzkin JL, Zhu N, Marier RL. Reliability of telemedicine examination. Telemed | | 997;3: | 4 |-57.

43. Alli PM, Ollayos CW, Thompson LD, Kapadia I, Butler DR, Williams BH, Rosenthal DL, O'leary T]. Telecytology: intraobserver and interobserver reproducibility in the diagnosis of cervical-vaginal smears. Hum Pathol 2001;32:1318-22.

44. Chorneyco K, Giesler R, Sabatino D, Ross C, Lobo F, Shuhaibar H, et al. Telepathology for routine light microscopic and frozen section diagnosis. Am J Clin Pathol 2002; I 17:783-90
45. Lee ES, Kim IS, Choi JS, Yeom BW, Kim HK, Han JH, et al. Accuracy and reproducibility of telecytology diagnosis of cervical smears. A tool for quality assurance programs. Am | Clin Pathol 2003; | | 9:356-60.

46. Stanford MR, Gras L, Wade A, Gilbert RE. Reliability of expert interpretation of retinal photographs for the diagnosis of toxoplasma retinochoroiditis. Br J Ophthalmol 2002;86:636-9.

47. Blomdahl S, Marén N, Lof R. Tele-ophthalmology for the treatment in primary care of disorders in the anterior part of the eye. J Telemed Telecare $2001 ; 7: 25-6$.

48. Declaração de Tel Aviv. Responsabilidades e normas éticas na utilização da telemedicina. In: 5 I a Assembléia Geral da Associação Médica Mundial em Tel Aviv [online]; 1999. [citado 2003 fev 25]. Disponível em: http:// www.unifesp.br/dis/set/law/declaracaotelaviv.html

49. Murdoch I. Telemedicine. Br J Ophthalmol I 999;83: I 254-6.

\section{www.ramb.org.br}

Em nosso site, você encontra as edições on line da Ramb com o mesmo conteúdo da revista impressa: as seções

À Beira do Leito, Comentário, Diretrizes em Foco, Panorama Internacional, Discussão de Caso, Imagem em Medicina,

Prática Clínica e ainda artigos originais.

Acesse www.ramb.org.br 